\title{
USO DE SOMBRAS NA CORREÇÃO DO EFEITO VINHETE
}

\author{
Using shadows to correct vignetting effects
}

NILTON NOBUHIRO IMAI ${ }^{1}$

AIRTON MARCO POLIDÓRIO ${ }^{2}$

ANTONIO MARIA GARCIA TOMMASELLI ${ }^{1}$

ANDERSON DE FREITAS BARROS ${ }^{1}$

${ }^{1}$ Universidade Estadual Paulista - UNESP

Presidente Prudente - SP - Brasil

${ }^{2}$ Universidade Estadual de Maringá - UEM

Maringá - PR - Brasil

nnimai@ fct.unesp.br; ampolido@din.uem.br; tomaseli@fct.unesp.br;

rioand@gmail.com

\section{RESUMO}

Variações do valor de brilho de alvos da superfície podem ocorrer no registro realizado por sensores imageadores. A variação desses valores de brilho que ocorrem em função da distância do pixel ao centro da imagem é conhecida como efeito vinhete e sua correção tem como objetivo oferecer um ajuste de brilho homogêneo na imagem. A proposta deste trabalho é apresentar uma metodologia para determinar um modelo de minimização desse efeito vinhete com base numa função ajustada pelo método dos mínimos quadrados, a partir dos valores de brilho de sombras. Uma das hipóteses adotadas é de que as variações de brilho registrados em pixels de sombra sejam adequadas para representar os efeitos do vinhete. Considerando que o efeito vinhete possa ser modelado como uma tendência de variação espacial dos valores de brilho da imagem foi realizada a análise de superfície de tendência de um conjunto de pixels amostrados em regiões de sombras. Foram adotadas duas abordagens para representar os valores de sombras que foram processados. A primeira, baseada nos componentes $\mathrm{R}, \mathrm{G}$ e $\mathrm{B}$ da imagem colorida; e a segunda estratégia, do componente I da imagem HSI. A fim de avaliar a metodologia realizou-se um estudo de caso com uma imagem aérea colorida. Os experimentos mostraram que o melhor resultado foi obtido pela aplicação do modelo nas componentes RGB, permitindo concluir que o efeito vinhete pode ser modelado com base em superfícies de tendência ajustadas aos valores RGB das 
sombras.

Palavras-chave: Sensoriamento Remoto; Efeito Vinhete; Análise de Variância; Superfície de Tendência.

\section{ABSTRACT}

When registering spectral radiance from surface targets, digital numbers recorded by the imagery sensor may vary. Such variation causes imperfections on the images coming from aerial surveys. Variation in the image brightness related to the distance from the center of the image is known as the vignetting effect. Correcting this effect aims at achieving an homogeneous image brightness. The purpose of this paper is to present a specific methodology to determine a model in order to minimize this vignette effect based on a model fit by Least Squares Method (LSM), using digital numbers (DN) from shadowed regions. The main hypothesis is that the recorded DN of shadow pixels should be suitable to model the vignetting effect. Considering that the vignetting effect could be modeled as a trend of spatial image variation, a trend surface analysis of a sample of pixels from shadowed regions was carried out. Two approaches were adopted to represent the shadow regions of an image. The first one takes into account the components $\mathrm{R}, \mathrm{G}, \mathrm{B}$ of the aerial image within the visible spectral band, and the second one considers the component I of the HSI image. In order to evaluate the methodology, a study case with a color aerial image was carried out. The findings showed that the best results were obtained by applying the model in the RGB components, which allows to conclude that the vignetting effect can be modeled based on trend surfaces fit on shadow regions DN.

Keywords: Remote Sensing; Vignetting Effect; Analysis of Variance; Trend Surface.

\section{INTRODUÇÃO}

Os elementos que compõem a superfície terrestre apresentam comportamento espectral muito variado e cada elemento pode apresentar uma curva de reflectância distinta, caracterizando um padrão de resposta espectral (POLIDORIO, 2007). As variações do fluxo de energia radiante refletido que são registrados em imagens digitais deveriam corresponder às variações da reflectância espectral dos alvos imageados. Entretanto, podem ocorrer variações nas respostas espectrais dos elementos registrados na cena, causando imperfeições nas imagens aéreas. Essas imperfeições ocorrem devido às influências de fenômenos de origem geométrica e atmosférica (LILLESAND e KIEFER, 1987).

Os fenômenos de origem geométrica estão associados à variação nos valores de brilho em função da distância do ponto ao centro da imagem, por exemplo, o efeito vinhete (LILLESAND e KIEFER, 1987). Esse efeito causa na imagem isolada uma distribuição não uniforme de iluminação (SILVA e CANDEIAS, 2009) e no mosaico, causa uma descontinuidade no brilho (NÓBREGA, 2002). 
Como o efeito vinhete influencia na iluminação da cena, a interpretação de elementos da superfície terrestre pode ser prejudicada, tornando necessária a correção desse efeito.

Uma das abordagens adotadas para estimar o efeito vinhete consiste em analisar imagens tomadas de uma cena cuja reflectância seja homogênea e a irradiância constante em toda região imageada (AGGARWAL et al., 2001; KIM e POLLEFEYS, 2008). Yu (2004) adota uma imagem de uma folha branca como referência. Com base nessa imagem, aplica técnicas de remoção de ruídos e uma transformada Wavelet a fim de extrair uma superfície suavizada que representa o efeito vinhete.

Zheng et al. (2009) propõem uma correção do efeito vinhete baseada nos dados de uma cena real, a qual é segmentada em regiões homogêneas. A estimativa baseiase nas variações que podem ser distinguidas entre as variações da cena das variações decorrentes do efeito vinhete. A abordagem adotada nesse trabalho também é baseada em cena real, mais especificamente uma imagem aérea.

Este trabalho propõe uma abordagem baseada na suposição de que o efeito vinhete produz uma variação do valor de brilho que pode ser representada por uma tendência contínua como de uma função polinomial, cujos parâmetros podem ser estimados a partir dos valores de brilhos em áreas de sombras. Do ponto de vista prático, sombras são comumente encontradas em imagens aéreas. Desta forma, adotou-se a hipótese de que as variações do valor de brilho registrado em sombras possam ser usadas como referência para ajustar um modelo de minimização do efeito vinhete. Essa hipótese baseia-se no fato de que as áreas de sombra representam regiões que não recebem energia solar diretamente, mas tão somente energia difusa proveniente da atmosfera e da vizinhança. Considera-se que a quantidade de energia difusa incidente seja desprezível e, ainda, que as variações decorrentes da energia difusa refletida também sejam aleatórias, por introduzir variações aleatórias que não fazem parte de uma tendência espacial esperada como resultado do efeito vinhete.

Desta forma, essa superfície pode ser capaz de minimizar o efeito vinhete presente na imagem. Assim como em Silva e Candeias (2009), a abordagem adotada baseia-se num modelo de superfície de compensação para representar os efeitos de variação de brilho numa cena, mas trata das diferenças de valores registrados relacionados principalmente ao efeito vinhete. Enquanto a proposta de Silva e Candeias (2009) procura tratar o conjunto de fenômenos que provocam as diferenças indesejáveis na cena, incluindo os efeitos relacionados com o Fator de Reflectância Bidirecional (FRB), na presente proposta o efeito do FRB não é considerado. Nesse caso, as variações decorrentes da geometria de iluminação e tomada da cena, relacionadas com a variação do FRB, produzem alterações de brilho em alvos que recebem energia solar diretamente da fonte. Desta forma, a abordagem adotada não é influenciada por esse fenômeno.

Vale destacar que a sombra é um tipo de alvo da cena que apresenta quatro aspectos favoráveis na estimativa de funções que representam o efeito vinhete: a) 
geralmente apresentam distribuição espacial que garante a presença de um elemento amostral em qualquer parte da cena; b) resposta espectral de valor baixo; c) como sua iluminação é indireta, a disponibilidade de energia incidente tem distribuição aleatória, pois só depende dos alvos da vizinhança e d) a energia registrada de regiões de sombra não sofre o efeito do FRB por não haver incidência de radiação direta sobre o alvo.

Assim, a distribuição espacial das variações de brilho, representada por um modelo polinomial foi obtido pela aplicação da técnica de mínimos quadrados com base na aplicação da regressão polinomial. O modelo polinomial é escolhido com base na análise de superfície de tendência (LANDIM, 1997) aplicada aos valores de sombras. Landim (1997) explica que a aplicação dessa análise permite “... separar dados mapeáveis em duas componentes: uma de natureza regional, representada pela própria superfície, e outra que revela as flutuações locais, representada pelos valores residuais". Desta forma, a componente de natureza regional pode representar as variações introduzidas pelo efeito vinhete, enquanto as flutuações locais estão associadas com a energia refletida pelos alvos que cercam cada sombra.

\subsection{Efeito Vinhete e Influência nas Imagens Aéreas}

$\mathrm{O}$ vinhete é o efeito radial de escurecimento da imagem, que escurece a borda e mantém clara a região central da imagem aérea (AMERICAN SOCIETY OF PHOTOGRAMMETRY, 1980). Trata-se de um efeito de diminuição do valor de radiância que depende da posição (YU, 2004).

$\mathrm{O}$ efeito vinhete pode ser óptico, natural e mecânico. $\mathrm{O}$ vinhete óptico depende do diâmetro da lente exposta, ou seja, é determinado pela abertura do diafragma. Quanto menor a abertura da lente, maior será o "f number" e menores serão os efeitos devidos ao vinhete (YU, 2004). O vinhete natural é próprio de cada sistema de lente e está associado à diminuição de iluminação que atinge o plano da imagem. $\mathrm{O}$ vinhete mecânico ocorre quando há obstáculos na região periférica da objetiva da câmara, por exemplo, com um para-sol.

No que se refere à abertura do obturador da câmara, Silva e Candeias (2009) afirmam que, para reduzir o arrastamento em voos para escala grande, uma abertura maior do obturador implica no aumento do efeito do vinhete óptico. Por outro lado, Nobrega (2002) diz que uma menor abertura do obturador implica na redução radial da intensidade de luz capturada pelo sistema de lentes.

De acordo com Nobrega (2002), a variação da intensidade de luz está associada à lei do cosseno descrita pelas relações geométricas formadas entre o ângulo de incidência dos raios luminosos, a distância focal da câmara e o plano do filme, como definido na equação a seguir.

$$
I(b)=I_{0} \cdot \cos (b)^{n}
$$


dado que: $\mathrm{I}_{0}$ é a iluminação para $b=0$ que atinge o plano do filme; $b$ é o ângulo entre o eixo óptico e o raio; e $n$ valor que varia de 2,5 a 4 .

Silva e Candeias (2009) afirmam que a utilização de acessórios, tais como extensão sobre a objetiva e filtro, agravam o efeito vinhete mecânico, pois contribuem para que os raios oblíquos não atinjam o ponto nodal.

\subsection{Correção do Efeito Vinhete}

Para Nóbrega e Quintanilha (2004), a correção do efeito vinhete pode ser realizada com balanceamento de cores e uniformização de histogramas entre as partes claras e escuras das imagens. Para Nóbrega (2002), muitos aplicativos comerciais utilizam estes procedimentos, porém os resultados nem sempre são satisfatórios.

Langhi e Tommaselli (2008) desenvolveram um programa em linguagem C que realiza correção radiométrica em bloco de imagens aéreas, pela análise dos histogramas e pelo ajuste de superfície parabolóide às diferenças radiométricas entre as imagens, que permite a correção do efeito vinhete.

Nobrega (2002) realizou testes no programa Erdas para verificar a eficiência desse programa quanto à correção do efeito vinhete. $\mathrm{O}$ autor apontou o uso de modelo de balanceamento por superfície na compensação da diferença no padrão de luminosidade das imagens. Porém, o modelo apresenta quatro superfícies que, mesmo adequando-se à maioria das necessidades, pode não ser suficiente como solução de balanceamento de imagens.

De acordo com Yu (2004), geralmente a correção do vinhete tem inicio com a tomada de uma imagem de uma cena na qual a irradiância seja constante. Assim como em Zheng et al. (2009), este trabalho propõe uma abordagem baseada na análise de cenas reais. O trabalho de Zheng et al. (2009) envolve um procedimento de classificação complexo. $\mathrm{Na}$ presente proposta as sombras presentes na cena constituem o tipo de alvo a serem amostradas, tornando a tarefa mais simples, inclusive devido ao fato de que a classificação desse tipo de alvo vem sendo aprimorado, como em Centeno e Pacheco (2011).

\section{METODOLOGIA DE TRABALHO}

A área de estudo mostrada na Figura (a) corresponde à região de área urbana coberta pela imagem aérea, na faixa do espectro do visível, obtida durante o voo realizado no município de Tarumã (SP) no dia 25/03/2007.

A imagem aérea foi adquirida pela câmara HASSELBLAD H2D do Sistema Aerotransportado de Aquisição e Pós-Processamento de Imagens Digitais (SAAPI), numa parceria entre a Universidade Estadual Paulista (UNESP) e a Empresa Engenharia, Mapeamento e Aerolevantamento Ltda. (ENGEMAP).

Para extrair o efeito vinhete da imagem é realizada uma análise dos níveis de cinza dos componentes $\mathrm{R}, \mathrm{G}$ e $\mathrm{B}$ das áreas de sombras, com base no distanciamento do centro da imagem. A correção do efeito vinhete é baseada num modelo polinomial que relaciona a diferença entre o valor livre do efeito vinhete e o valor 
registrado de um pixel na cena, com a posição desse pixel na cena, obtido por regressão. A análise da distribuição espacial dos valores que representam sombras é realizada nos espaços de atributos de cor RGB e HSI. Nesse segundo caso, são analisadas as variações da componente I, uma vez que se espera que o efeito vinhete provoque alterações no brilho de cada pixel, conforme sua posição na cena.

Polinômios de primeiro grau, bilinear, de segundo grau e cúbico foram ajustados e avaliados com aplicação da Análise de Variância (ANOVA).

Os valores de ND (número digital) constituem um conjunto de elementos amostrais selecionados, manualmente (no caso 265 elementos), com base em análise visual. Adotou-se como critério selecionar o ponto mais central de cada sombra. Cada elemento amostral possui as posições L (linha) e C (coluna) do pixel de cada elemento de sombra na imagem e os valores dos componentes R, G, B e I do pixel de sombra. Esses valores R, G e B da amostra foram extraídos com o Matlab e o valor da componente I calculado com base nesses valores de atributos do pixel.

Após a coleta de dados, foi realizado o ajuste de cada um dos polinômios mencionados, pelo método dos mínimos quadrados (MMQ) para representar o fenômeno. Em seguida foi realizada a análise de variância para avaliar os modelos de polinômios. Neste caso, outras estatísticas não são examinadas, uma vez que o objetivo desse processo é modelar somente a tendência espacial de variação de brilho dessas sombras.

A análise de variância foi aplicada para comparar os modelos ajustados com base na contribuição dos sucessivos coeficientes parciais de regressão e fornecer uma medida de qualidade do ajustamento aos dados devido a cada um dos incrementos da equação polinomial. Essa comparação tem como finalidade verificar qual modelo representa melhor uma possível tendência da distribuição espacial do fenômeno representado (LANDIM, 1997). Nesse sentido é utilizada a Tabela ANOVA conforme o preenchimento mostrado na Tabela 1.

Parte dos valores necessários ao preenchimento da tabela ANOVA foram processados nos programas Surfer e Excel. Os valores de resíduos e os parâmetros de cada polinômio são obtidos no programa Surfer. As operações de cálculos referentes à soma dos quadrados, média dos quadrados e outras operações são realizadas no programa Excel.

Com base na Distribuição F de Fisher Snedecor, cada polinômio deve ser avaliado num teste baseado no valor $\mathrm{F}$ calculado $(\mathrm{Fc})$, o qual é comparado com o valor de $\mathrm{F}$ crítico $(\mathrm{Ft})$, de acordo com o número de graus de liberdade do polinômio e dos resíduos. Esse teste foi realizado com $5 \%$ de significância, sendo que todos polinômios aceitos no teste foram comparados entre si com base no incremento da contribuição do grau de explicação obtido na mudança de um polinômio de menor grau para outro de maior grau. Os testes aplicados são descritos em seguida. 
Tabela 1 - Análise da variância para seleção do melhor polinômio. FONTE: adaptada de Landim (1998).

\begin{tabular}{|c|c|c|c|c|}
\hline $\begin{array}{l}\text { Fonte de } \\
\text { Variação } \\
\end{array}$ & \begin{tabular}{|c|} 
Soma dos \\
Quadrados \\
\end{tabular} & $\begin{array}{c}\text { Grau de } \\
\text { Liberdade }\end{array}$ & $\begin{array}{l}\text { Média dos } \\
\text { Quadrados } \\
\end{array}$ & $\mathbf{F}_{\mathrm{C}}$ \\
\hline Regressão de grau $p$ & SQP & $\mathrm{k}$ & $\mathrm{MQP}=\mathrm{SQP} / \mathrm{k}$ & \multirow[b]{2}{*}{ MQP/MQR } \\
\hline $\begin{array}{c}\text { Resíduos referentes à } \\
\text { p }\end{array}$ & SQR & $n-k-1$ & $\mathrm{MQR}=\mathrm{SQR} /(\mathrm{n}-\mathrm{k}-1)$ & \\
\hline $\begin{array}{c}\text { Regressão de grau } \\
\text { p+1 }\end{array}$ & SQP1 & $\mathrm{m}$ & $\mathrm{MQP} 1=\mathrm{SQP} 1 / \mathrm{m}$ & \multirow{2}{*}{$\begin{array}{c}\mathrm{MQP1/MQR} \\
1\end{array}$} \\
\hline $\begin{array}{c}\text { Resíduos referentes à } \\
\mathrm{p}+1 \\
\end{array}$ & SQR1 & n-m-1 & $\mathrm{MQR} 1=\mathrm{SQR} /(\mathrm{n}-\mathrm{m}-1)$ & \\
\hline $\begin{array}{c}\text { Incremento de } \mathrm{p} \text { para } \\
\mathrm{p}+1\end{array}$ & SQI & $\mathrm{m}-\mathrm{k}$ & MQI=SQI/(m-k) & \multirow[t]{2}{*}{ MQI/MQR1 } \\
\hline Total & SQT & $n-1$ & $\mathrm{MQT}=\mathrm{SQT} /(\mathrm{n}-1)$ & \\
\hline
\end{tabular}

onde:

variação total: $S Q T=\sum y_{i}^{2}-\left\lfloor\left(\sum y_{i}\right)^{2} / n\right\rfloor$;

variação devida à superfície calculada: $S Q P=\sum y_{i}^{\prime 2}-\left(\left(\sum y_{i}^{\prime}\right)^{2} / n\right]$;

variação devida aos resíduos: $S Q R=S Q T-S Q P$;

variação devida ao incremento: $S Q I=S Q P 1-S Q P$;

teste de significância relativo à superfície de tendência de grau p: $M Q P / M Q R$;

teste de significância relativo à superfície de tendência de grau p+1: $M Q P 1 / M Q R 1$;

teste de significância relativo à melhoria de ajuste da superfície $\mathrm{p}+1$ comparada a p: $M Q I / M Q R 1$;

SQ: soma dos quadrados; MQ: média quadrática;

$\mathrm{F}_{\mathrm{C}}$ : razão entre médias quadráticas;

n: número de observações;

$\mathrm{k}$ : coeficientes da regressão polinomial de grau p;

$\mathrm{m}$ : coeficientes da regressão polinomial de grau $\mathrm{p}+1$;

y e y': valor da variável observada e estimada pela regressão polinomial, respectivamente.

A hipótese nula $\left(\mathrm{H}_{0}\right)$ significa que a contribuição do incremento polinomial para o ajuste aos dados é nula e a hipótese alternativa $\mathrm{H}_{1}$ significa que a contribuição do incremento polinomial para o ajuste aos dados é significativa.

Portanto, se o valor de Fc (calculado) for menor que Ft (tabelado), aceita-se a hipótese nula $\mathrm{H}_{0}$, pois o incremento do grau de explicação ou da diminuição da variância pela adoção do modelo analisado é insignificante, no nível de 5\%, para 
adotar o modelo analisado como superfície de tendência. Caso contrário, rejeita-se a hipótese nula $\mathrm{H}_{0}$ e se aceita a hipótese alternativa $\mathrm{H}_{1}$.

Depois de encontrado o polinômio que representa melhor a tendência dos valores de brilho das sombras no espaço, aplica-se a correção do efeito vinhete com base na superfície de tendência selecionada, a partir de procedimentos realizados no programa Matlab. Esses procedimentos são descritos a seguir.

Produz-se uma superfície de compensação a qual é representada por uma matriz com o mesmo número de linhas e colunas da imagem que deve ser corrigida do efeito vinhete, a qual deve ser produzida como função do modelo de tendência selecionado. O valor máximo encontrado nessa matriz é subtraído pelo valor de cada elemento dessa matriz. O resultado dessa operação gera uma matriz com estimativas do valor de correção do efeito vinhete. Os elementos mais próximos ao centro dessa matriz devem ser valores baixos e os elementos mais distantes serão valores mais altos. Por fim, essa matriz é adicionada à imagem original, nesse caso, à imagem com vinhete.

Essa abordagem é similar ao processo de minimização da componente de tendência adotados em análise de séries temporais. Mais detalhes são encontrados em Morettin (2004).

\section{RESULTADOS E DISCUSSÃO}

Os experimentos para correção do efeito vinhete foram realizados numa imagem da faixa do espectro do visível, do Município de Tarumã, como pode ser visto na Figura 1. Essa imagem foi tomada por uma câmara Hasselblad do sistema SAAPI (RUY et al, 2008; RUY et al, 2012).

Figura 1 - (a) Imagem original. (b) Imagem corrigida pelas componentes RGB.

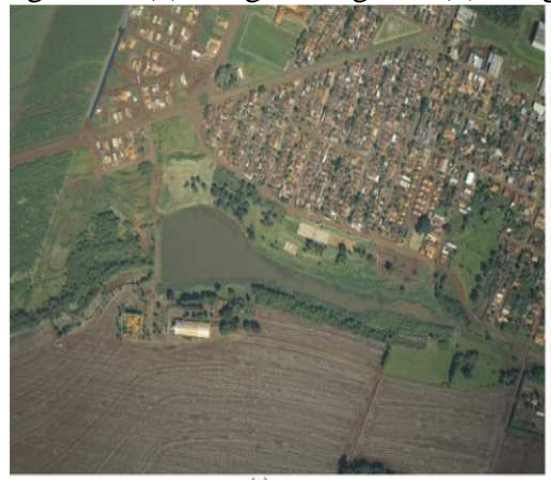

(a)

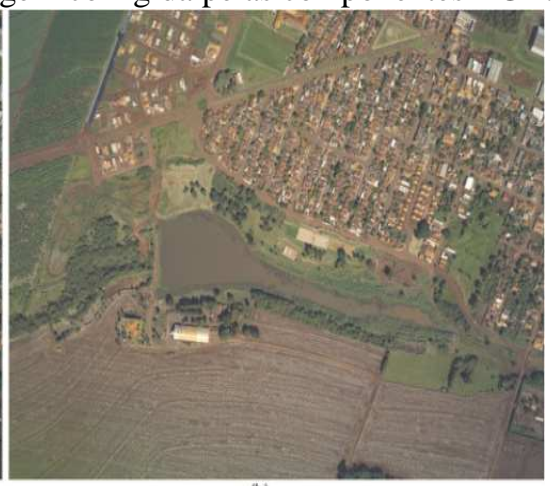

(b)

A Figura 2 representa a variação dos valores de brilho, ND, das componentes espectrais correspondentes às cores azul, verde e vermelho de pixels de sombra em função da distância do pixel ao centro da cena. 
Figura 2 - ND das componentes RGB de pixels de sombras da imagem original em relação a sua distância ao centro da imagem.

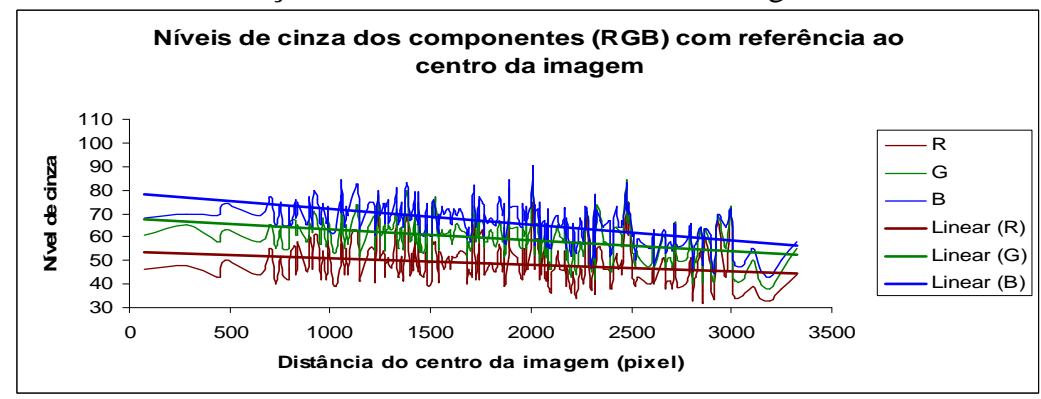

O gráfico apresentou valores de brilho elevados nas sombras mais próximas do centro da imagem e à medida que as sombras se afastam do centro da imagem há um decréscimo nos valores de brilho das sombras.

Observou-se que as linhas de tendência aplicadas aos componentes RGB apresentam inclinações muito acentuadas. Cada polinômio foi testado através da análise de variância. Todos apresentaram valores de Fc maiores que zero.

A Tabela 2 mostra os resultados obtidos nos testes de avaliação dos polinômios: linear, bilinear, quadrático e cúbico, realizados de acordo com a formulação apresentada na Tabela1. Os valores das médias dos quadrados obtidos e correspondentes valores de $\mathrm{F}$ calculado - Fc, para cada polinômio e utilizados nos testes de hipótese, estão apresentados nos dois últimos campos.

Tabela 2 - Valores calculados para avaliação dos polinômios dos componentes R, $\mathrm{G}, \mathrm{B}$ e I.

\begin{tabular}{|c|c|c|c|c|c|}
\hline Componente & $\begin{array}{l}\text { Fonte de } \\
\text { Variação }\end{array}$ & $\begin{array}{c}\text { Soma dos } \\
\text { Quadrados }\end{array}$ & $\begin{array}{c}\text { Grau de } \\
\text { Liberdade }\end{array}$ & $\begin{array}{l}\text { Média dos } \\
\text { Quadrados }\end{array}$ & Fc \\
\hline \multirow{12}{*}{$\mathrm{R}$} & Linear & 8260,298359 & 2 & 4130,149180 & \multirow{3}{*}{142,891232} \\
\hline & Resíduos & 7572,886546 & 262 & 28,904147 & \\
\hline & Total & 15833,184906 & 264 & 59,974185 & \\
\hline & Bilinear & 9319,989175 & 3 & 3106,663058 & \multirow{3}{*}{124,491738} \\
\hline & Resíduos & 6513,195731 & 261 & 24,954773 & \\
\hline & Total & 15833,184906 & 264 & 59,974185 & \\
\hline & Quadrática & 10473,251612 & 5 & 2094,650322 & \multirow{3}{*}{101,216639} \\
\hline & Resíduos & 5359,933294 & 259 & 20,694723 & \\
\hline & Total & 15833,184906 & 264 & 59,974185 & \\
\hline & Cúbica & 10975,502193 & 9 & 1219,500244 & \multirow{3}{*}{64,016648} \\
\hline & Resíduos & 4857,682713 & 255 & 19,049736 & \\
\hline & Total & 15833,184906 & 264 & 59,974185 & \\
\hline \multirow[t]{2}{*}{$G$} & Linear & 7985,726322 & 2 & 3992,863161 & \multirow{2}{*}{143,025546} \\
\hline & Resíduos & 7314,288772 & 262 & 27,917133 & \\
\hline
\end{tabular}

Bol. Ciênc. Geod., sec. Artigos, Curitiba, v. 20, nº 1, p.100-116, jan-mar, 2014. 


\begin{tabular}{|c|c|c|c|c|c|}
\hline & Total & 15300,015094 & 264 & 57,954603 & \\
\hline & Bilinear & 8127,716758 & 3 & 2709,238919 & \multirow{3}{*}{98,589228} \\
\hline & Resíduos & 7172,298336 & 261 & 27,480070 & \\
\hline & Total & 15300,015094 & 264 & 57,954603 & \\
\hline & Quadrática & 10821,555794 & 5 & 2164,311159 & \multirow{3}{*}{125,167285} \\
\hline & Resíduos & 4478,459300 & 259 & 17,291349 & \\
\hline & Total & 15300,015094 & 264 & 57,954603 & \\
\hline & Cúbica & 10923,543855 & 9 & 1213,727095 & \multirow{3}{*}{70,719169} \\
\hline & Resíduos & 4376,471240 & 255 & 17,162632 & \\
\hline & Total & 15300,015094 & 264 & 57,954603 & \\
\hline \multirow{12}{*}{ B } & Linear & 6559,251044 & 2 & 3279,625522 & \multirow{3}{*}{87,405000} \\
\hline & Resíduos & 9830,809334 & 262 & 37,522173 & \\
\hline & Total & 16390,060377 & 264 & 62,083562 & \\
\hline & Bilinear & 6567,739654 & 3 & 2189,246551 & \multirow{3}{*}{58,172948} \\
\hline & Resíduos & 9822,320723 & 261 & 37,633413 & \\
\hline & Total & 16390,060377 & 264 & 62,083562 & \\
\hline & Quadrática & 11555,817798 & 5 & 2311,163560 & \multirow{3}{*}{123,823195} \\
\hline & Resíduos & 4834,242579 & 259 & 18,665029 & \\
\hline & Total & 16390,060377 & 264 & 62,083562 & \\
\hline & Cúbica & 11944,416515 & 9 & 1327,157391 & \multirow{3}{*}{76,125111} \\
\hline & Resíduos & 4445,643862 & 255 & 17,433897 & \\
\hline & Total & 16390,060377 & 264 & 62,083562 & \\
\hline \multirow{12}{*}{ I } & Linear & 0,166909 & 2 & 0,083455 & \multirow{3}{*}{157,525906} \\
\hline & Resíduos & 0,138803 & 262 & 0,000530 & \\
\hline & Total & 0,305712 & 264 & 0,001158 & \\
\hline & Bilinear & 0,178288 & 3 & 0,059429 & \multirow{3}{*}{121,726941} \\
\hline & Resíduos & 0,127425 & 261 & 0,000488 & \\
\hline & Total & 0,305712 & 264 & 0,001158 & \\
\hline & Quadrática & 0,219270 & 5 & 0,043854 & \multirow{3}{*}{131,395753} \\
\hline & Resíduos & 0,086442 & 259 & 0,000334 & \\
\hline & Total & 0,305712 & 264 & 0,001158 & \\
\hline & Cúbica & 0,226858 & 9 & 0,025206 & \multirow{3}{*}{81,512628} \\
\hline & Resíduos & 0,078854 & 255 & 0,000309 & \\
\hline & Total & 0,305712 & 264 & 0,001158 & \\
\hline
\end{tabular}

A escolha do polinômio que representa a superfície de tendência para cada um dos componentes R, G, B e I foi realizada com base na análise de variância ANOVA, aplicada para comparar a diferença entre as contribuições entre um par de polinômios. Nesse sentido, foram calculados os valores de F do incremento obtido por um polinômio mais complexo em relação a outro mais simples. Na Tabela 3 estão apresentados os valores de $\mathrm{F}$ calculados e tabelados para representar o aumento da contribuição do grau de explicação do fenômeno por um polinômio 
mais complexo em relação a outro mais simples para o caso da componente R. Os cálculos foram realizados de acordo com a formulação da Tabela 1.

Tabela 3 - Análise de incrementos entre polinômios para componente R.

\begin{tabular}{c|c|c}
\hline Fonte de Variação & Fc & Ft \\
\hline Incremento Bilinear-Linear & 42,464454 & 3,881 \\
\hline Incremento Quadrática-Bilinear & 27,863684 & 3,033 \\
\hline Incremento Cúbica-Quadrática & 6,591306 & 2,409 \\
\hline
\end{tabular}

Os valores de Ft correspondem aos valores críticos no nível de $5 \%$ de probabilidade da distribuição $F$ de Fisher-Snedecor. Nas três comparações apresentadas na Tabela 3, o valor de Fc devido ao incremento do polinômio de menor grau para o polinômio de maior grau ser maior que Ft, rejeita-se a hipótese nula $\mathrm{H}_{0}$ e aceita-se a alternativa $\mathrm{H}_{1}$, de que é significativa a diferença dada pelo incremento devido à adoção de uma superfície de tendência de maior ordem polinomial. Nesse caso, os resultados mostram que os dados do componente R são representados adequadamente por uma superfície de tendência cúbica.

Na Tabela 4 estão apresentados os valores de Fc calculados para comparar os polinômios ajustados para a componente G. Nesse caso, os testes indicam que, no nível de significância de 5\%, o polinômio que representa a tendência espacial de variação dos valores é de segunda ordem.

Tabela 4 - Análise de incrementos entre polinômios para componente G.

\begin{tabular}{c|c|c}
\hline Fonte de Variação & Fc & Ft \\
\hline Incremento Bilinear-Linear & 5,167033 & 3,881 \\
\hline Incremento Quadrática-Bilinear & 77,895573 & 3,033 \\
\hline Incremento Cúbica-Quadrática & 1,485612 & 2,409 \\
\hline
\end{tabular}

Os valores de Fc do incremento entre polinômios de maior grau com o de menor grau, ajustados para as observações da componente B, que foram aceitos pela aplicação da ANOVA em relação a situação na qual não há indícios de tendência espacial (média) estão apresentados na Tabela 5.

Tabela 5 - Análise de incrementos entre polinômios para componente B.

\begin{tabular}{c|c|c}
\hline Fonte de Variação & Fc & Ft \\
\hline Incremento Bilinear Linear & 0,225560 & 3,881 \\
\hline Incremento Quadrática Linear & 89,232234 & 2,642 \\
\hline Incremento Cúbica Quadrática & 5,572459 & 2,409 \\
\hline
\end{tabular}

Com base nos resultados mostrados na Tabela 5, seleciona-se a superfície de tendência cúbica para representar a variação espacial da componente B. 
Da mesma forma que a análise foi conduzida para comparar os polinômios ajustados para as componentes RGB, realizou-se uma comparação dos polinômios ajustados que foram aceitos para representar a tendência espacial da componente I. Os resultados dessa comparação estão apresentados na Tabela 6 , sendo que a superfície de tendência de variação espacial indicado pelos testes, com grau de 5\% de significância, foi o polinômio cúbico.

Tabela 6 - Análise de incrementos entre polinômios para componente I.

\begin{tabular}{c|c|c}
\hline Fonte de Variação & Fc & Ft \\
\hline Incremento Bilinear Linear & 23,306144 & 3,881 \\
\hline Incremento Quadrática Linear & 61,395703 & 2,642 \\
\hline Incremento Cúbica Quadrática & 6,134517 & 2,409 \\
\hline
\end{tabular}

Os coeficientes dos polinômios selecionados pela aplicação da ANOVA estão apresentados na Tabela 7.

Tabela 7 - Coeficientes e polinômios dos componentes R, G, B e I.

\begin{tabular}{|c|c|c|c|c|}
\hline \multicolumn{5}{|c|}{ Regressão Superfície } \\
\hline \multicolumn{5}{|c|}{$\begin{array}{c}\text { Equação Quadrática } \\
\mathrm{Z}(\mathrm{X}, \mathrm{Y})=\mathrm{A}_{00}+\mathrm{A}_{01} \mathrm{Y}+\mathrm{A}_{02} \mathrm{Y}^{2}+\mathrm{A}_{10} \mathrm{X}+\mathrm{A}_{11} \mathrm{XY}+\mathrm{A}_{20} \mathrm{X}^{2}\end{array}$} \\
\hline \multicolumn{5}{|c|}{$\begin{array}{c}\text { Equação Cúbica } \\
\mathrm{Z}(\mathrm{X}, \mathrm{Y})=\mathrm{A}_{00}+\mathrm{A}_{01} \mathrm{Y}+\mathrm{A}_{02} \mathrm{Y}^{2}+\mathrm{A}_{03} \mathrm{Y}^{3}+\mathrm{A}_{10} \mathrm{X}+\mathrm{A}_{11} \mathrm{XY}+\mathrm{A}_{12} \mathrm{XY}^{2}+\mathrm{A}_{20} \mathrm{X}^{2}+ \\
\mathrm{A}_{21} \mathrm{X}^{2} \mathrm{Y}+\mathrm{A}_{30} \mathrm{X}^{3}\end{array}$} \\
\hline $\begin{array}{c}\text { Coefici } \\
\text { ente }\end{array}$ & $\begin{array}{c}\text { Cúbica } \\
\text { (Componente R) }\end{array}$ & $\begin{array}{c}\text { Quadrática } \\
\text { (Componente G) }\end{array}$ & $\begin{array}{c}\text { Cúbica } \\
\text { (Componente B) }\end{array}$ & $\begin{array}{c}\text { Cúbica } \\
(\text { Componente I) }\end{array}$ \\
\hline $\mathrm{A}_{00}$ & 65,515420907866 & 64,903625683283 & 62,113004902524 & 0,3031070497209 \\
\hline $\mathrm{A}_{01}$ & $-0,01105934435$ & $-0,0004929859117$ & 0,0046750368534 & $-4,74229000 \mathrm{E}-05$ \\
\hline $\mathrm{A}_{02}$ & $-8,80047508 \mathrm{E}-07$ & $-1,5777965265 \mathrm{E}-06$ & $-3,833738932 \mathrm{E}-06$ & $-1,18022567 \mathrm{E}-09$ \\
\hline $\mathrm{A}_{03}$ & $6,056729355 \mathrm{E}-10$ & $\mathrm{NE}$ & $7,0165431234 \mathrm{E}-10$ & $1,547818756 \mathrm{E}-12$ \\
\hline $\mathrm{A}_{10}$ & 0,0001012894280 & 0,00346443903571 & 0,00998950613728 & $-4,47756448 \mathrm{E}$ \\
\hline $\mathrm{A}_{11}$ & $4,22377784110 \mathrm{E}-06$ & $8,8180664135 \mathrm{E}-07$ & $-2,588297445 \mathrm{E}-07$ & $2,049326896 \mathrm{E}-08$ \\
\hline $\mathrm{A}_{12}$ & $-9,1402825407 \mathrm{E}-10$ & $\mathrm{NE}$ & $-5,725251225 \mathrm{E}-10$ & $-3,50423974 \mathrm{E}-12$ \\
\hline $\mathrm{A}_{20}$ & $-1,6199737408 \mathrm{E}-06$ & $-1,2598099034 \mathrm{E}-06$ & $-2,156593457 \mathrm{E}-06$ & $-4,04944134 \mathrm{E}-09$ \\
\hline $\mathrm{A}_{21}$ & 4,54008924967E-11 & $\mathrm{NE}$ & $4,0589910665 \mathrm{E}-10$ & $-5,51003562 \mathrm{E}-13$ \\
\hline $\mathrm{A}_{30}$ & $9,37429328927 \mathrm{E}-11$ & $\mathrm{NE}$ & $-2,773456476 \mathrm{E}-11$ & $3,395717050 \mathrm{E}-14$ \\
\hline
\end{tabular}

Os coeficientes que receberam NE (Não Existe) correspondem aos coeficientes que na equação quadrática não existem.

As variáveis independentes $\mathrm{Y}$ e $\mathrm{X}$ são coordenadas da posição de um elemento, as quais estão de acordo com a configuração do programa Matlab. $\mathrm{O}$ elemento $\mathrm{Y}$ representa a posição $\mathrm{L}$ (linha) na imagem, com origem no canto 
superior esquerdo e crescente para baixo. $\mathrm{O}$ elemento $\mathrm{X}$ representa a posição $\mathrm{C}$ (coluna) na imagem, com origem no canto superior esquerdo e crescente para a direita, sendo que $\mathrm{Y}$ e $\mathrm{X}$ são os números do conjunto $\mathrm{Z}^{+}$, excluindo o zero, ou seja, $\mathrm{Z}^{*+}=\{1,2,3,4, \ldots\}$.

Em ambas abordagens, o resultado final foi a imagem ajustada pela aplicação de superfícies de compensação. Essas superfícies de compensação são matrizes com o mesmo número de linhas e colunas da imagem e são calculadas com base nos polinômios selecionados pela ANOVA. Cada polinômio é usado para produzir uma matriz que representa uma imagem de um cenário no qual a irradiância do alvo imageado é constante, perturbada pelo efeito vinhete. Sendo assim, uma matriz que representa uma superfície de compensação é formada pelos valores da diferença entre o valor de cada pixel dessa imagem alterada pelo vinhete e seu valor máximo.

A Figura 1(a) apresenta a imagem colorida original e a Figura 1(b) a imagem corrigida com esse tipo de superfície de compensação aplicada aos componentes RGB (primeira abordagem). O gráfico da Figura mostra os valores de brilho - ND de cada componente RGB dos elementos amostrais de sombras em relação à distância ao centro da imagem. O gráfico da Figura 3 apresentou tendência dos ND das sombras quase constantes, conforme sua distância ao centro da imagem. Observa-se que as linhas de tendência da Figura 2, com inclinações negativas muito acentuadas, foram corrigidas após a aplicação da correção do vinhete aplicado nas componentes RGB, como pode ser visto na Figura 3.

Figura 3 - ND das componentes RGB de pixels de sombras da imagem corrigida pelas componente RGB, em relação a sua distância ao centro da imagem.

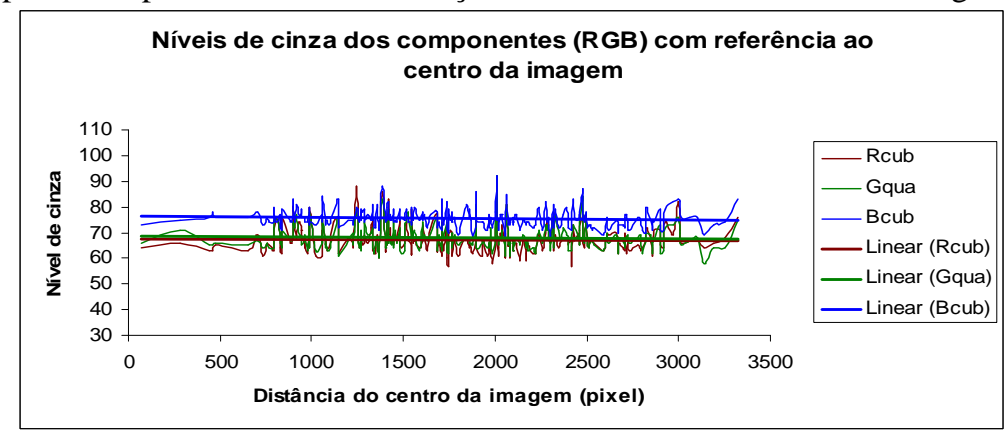

A Figura 4(b) apresenta a imagem produzida pela correção obtida com a aplicação da superfície de compensação na componente I (segunda abordagem). Nesse caso, após aplicar o ajuste no componente I, a imagem HSI foi convertida em imagem RGB. Ao lado dessa figura, está novamente representada a imagem original Figura 4(a). 
Figura 4 - (a) Imagem original. (b) Imagem corrigida pela componente I.

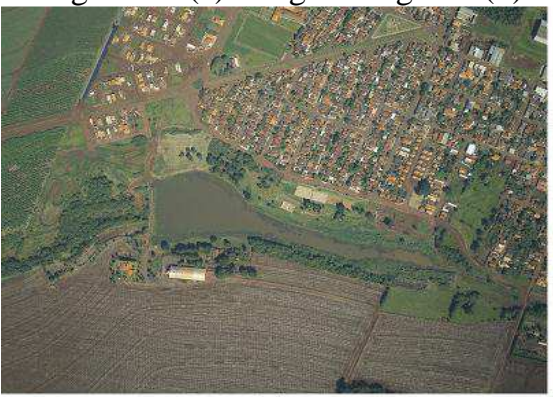

(a)

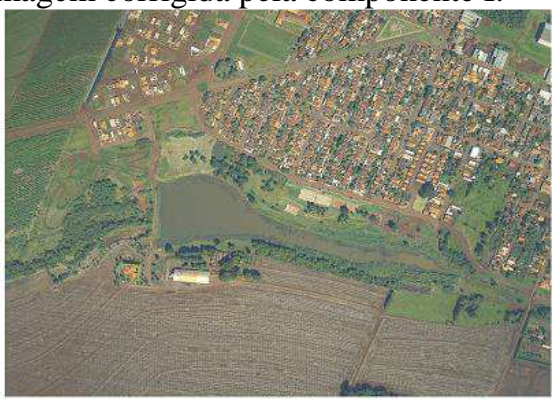

(b)

Dessa imagem corrigida, foram extraídos os ND de RGB para produzir o gráfico da Figura 5 e verificar a qualidade da correção do efeito vinhete. O gráfico da Figura 5 mostra que os ND das sombras, à medida que se afastam do centro da imagem foram corrigidos do efeito de escurecimento provocado pelo vinhete. Podese observar que as linhas de tendência com inclinações negativas muito acentuadas passaram a apresentar menor grau de inclinação após a aplicação da correção do efeito vinhete aplicada na componte I.

Figura 5 - ND das componentes RGB de pixels de sombras da imagem corrigida pela componente I, em relação a sua distância ao centro da imagem.

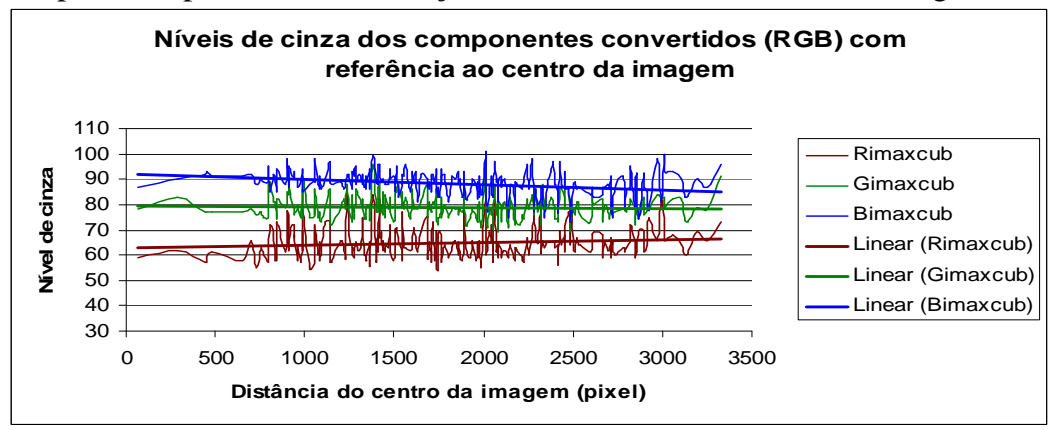

Uma comparação visual dos gráficos indica que a aplicação da correção em cada componente RGB minimiza melhor a tendência devido ao vinhete do que a correção baseada na componente de intensidade. Apesar disso, a correção aplicada na componente intensidade também minimizou as tendências de variação espacial do ND dos pixels de sombras mas não introduziu alteração das diferenças entre os valores médios de cada componente RGB como no caso da abordagem anterior. Alterações de valores médios não prejudicam a qualidade do produto pois basta aplicar uma correção radiométrica para recuperar o valor original. 


\section{CONCLUSÕES}

Neste trabalho foi proposta uma técnica para correção do efeito vinhete com aplicação de uma superfície de tendência. A avaliação experimental mostrou que esta técnica apresentou resultados adequados para produção de mosaicos ou mesmo como produto para análises posteriores.

Os resultados obtidos confirmam a hipótese de que os valores de brilhos das sombras podem oferecer informações para produzir uma superfície capaz de corrigir o efeito vinhete pela compensação da diferença no padrão de luminosidade da imagem.

A abordagem da primeira estratégia para corrigir o efeito vinhete, baseado na correção das componentes RGB, produziu resultado capaz de minimizá-lo. O decréscimo nos ND das sombras conforme aumenta sua distância ao centro da imagem, observado no gráfico da Figura 2, foi minimizado com essa abordagem, como pode ser verificado no gráfico da Figura 3. As inclinações muito acentuadas das linhas de tendência observadas no gráfico da Figura 1 foram minimizadas após aplicação da primeira estratégia.

A segunda abordagem para corrigir o efeito vinhete também produziu resultado capaz de minimizá-lo. O decréscimo nos ND das sombras, observado no gráfico da Figura 2, foi minimizado com essa abordagem, como verificado no gráfico da Figura 5.

As linhas de tendência com inclinações muito acentuadas, observadas no gráfico da Figura 2 foram minimizadas após a aplicação da correção da componente de intensidade.

A correção realizada em cada uma das componentes produziu um resultado cujas sombras possuem ND mais constantes conforme varia a posição da sombra, do que a correção aplicada na componente de intensidade. Nota-se que, apesar da componente intensidade sintetizar as variações do ND dos canais que registram as bandas do visível, a correção aplicada individualmente, em cada componente RGB, ainda produz resultado com menor grau de inclinação da tendência linear em relação a distância das sombras ao centro da imagem.

Por fim, recomenda-se para trabalhos futuros a aplicação desta abordagem em um conjunto de dados de sombras, coletado automaticamente, para comparar os resultados e permitir o avanço nos estudos da correção do efeito vinhete.

\section{AGRADECIMENTOS}

À Coordenação de Aperfeiçoamento de Pessoal de Nível Superior pelo auxilio financeiro destinado a essa pesquisa sob a forma de bolsa de estudo e à Engemap pelo fornecimento das imagens utilizadas.

\section{REFERÊNCIAS BIBLIOGRÁFICAS}

AGGARWAL, M.; HUA, H.; AHUAJA, N. 2001. On cosine-fourth and vignetting effects in real lenses. IEEE Eighth International Conference on Computer Vision, ICCV, 2001. Proceedings. V.2, 472-479, 2001. 
AMERICAN SOCIETY OF PHOTOGRAMMETRY. Manual of photogrammetry. 4. ed. Falls Church, VA: Chester C., 1980. 1056 p.

CENTENO, J.; PACHECO, A. Classificação de sombra em imagens orbitais e aéreas utilizando uma matriz de co-ocorrência modificada. Boletim de Ciências Geodésicas, (17) 1: 75-92, 2011.

GROSSBERG, M.D.; NAYAR, S.K. Determining the camera response from images: what is knowable? IEEE Transactions on Pattern Analysis and Machine Intelligence. (25): 11, 1455- 1467, 2003.

JUANG, R.; MAJUMDER, A. Photometric Self-Calibration of a Projector-Camera System. IEEE $25^{\text {th }}$ Conference on Computer Vision and Pattern Recognition, 2007. CVPR '07. , (1): 1-8, 2007.

KIM, S. J. and POLLEFEYS, M. Robust Radiometric Calibration and Vignetting Correction. IEEE Transactions on Pattern Analysis and Machine Intelligence (30): 4, $562-576,2008$.

LANDIM, P. M. B. Análise estatística de dados geológicos. São Paulo: EDUNESP, 2008. 226 p.

LANGHI, P.; TOMMASELLI, A. M. G. Correção radiométrica de imagens aéreas digitais por meio de ajuste polinomial. Simpósio Brasileiro de Ciências Geodésicas e Tecnologias da Geoinformação. Anais, UFPE, 2008.

LILLESAND, T.M.; KIEFER, R.W. Remote Sensing and Image Interpretation. 2. ed. New York: John Wiley \& Sons, 1987. 721p.

MORETTIN, P. A.; TOLOI, C. M. C. Análise de séries temporais. São Paulo: Edgard Blücher, 2004. 535 p.

NÓBREGA, R. A. A. Análise do Balanceamento de imagens aplicado a Fotogrametria. 2002. 102 p. Dissertação (Mestrado em Engenharia de Transportes). Escola Politécnica. Universidade de São Paulo. São Paulo, 2002.

NÓBREGA, R. A. A; QUINTANILHA J. A. Comparative Analysis of Automatic Digital Image Balancing and Standard Histogram Enhancement Techniques in Remote Sensing Imagery. Revista Brasileira de Cartografia. (01): 56, 55-64, 2004.

POLIDORIO, A. M. Detecção de elementos da paisagem urbana em imagens aéreas multiespectrais. 2007. 178 f. Tese (Doutorado em Ciências Cartográficas) - Universidade Estadual Paulista, Faculdade de Ciências e Tecnologia, Presidente Prudente, 2007.

RUY, R. da S., TOMMASELli, A. M. G, GALO, M., HASEGAWA, J. K., MENOSSI, R. C. Fototriangulação com parâmetros adicionais para câmaras digitais: uma avaliação experimental. Boletim de Ciências Geodésicas. (14):4, $571-587,2008$.

RUY, R. da S., TOMMASELLI, A. M. G, GALO, M., HASEGAWA, J. K., REIS, T. T. Acuracy analysis of modular aerial digital system SAAPI in projects of large areas In: EuroCow2012 - International Calibration and Orientation Workshop, 2012, Castelldefels. Proceedings of Eurocow 2012. Castelldefels: ISPRS, 2012. v.1. p.1 - 6. 
SILVA, D. C.; CANDEIAS, A. L. B. Causas da iluminação não uniforme em fotografias aéreas coloridas. Revista Brasileira de Cartografia. V. 2. N. 61. p. 105-115, 2009.

YU, WONPIL. Practical anti-vignetting methods for digital cameras. IEEE Transactions on Consumer Electronics . V. 50. N. 4. p. 975 - 983, 2004.

ZHENG, Y.; LIN, S.; KAMBHAMETTU, C.; YU, J.; KANG, S. B. Single-Image Vignetting Correction. IEEE Transactions on Pattern Analysis and Machine Intelligence. V. 31. N. 12. p. 2243 - 2256, 2009.

(Recebido em setembro de 2011. Aceito em outubro de 2013). 